\title{
Evaluación de la calidad tecnológica, nutricional y sensorial de productos de panadería por sustitución de harina de trigo por harina integral de arroz
}

\author{
Evaluation of the technological, nutritional and sensory qualities of bakery products \\ produced with partial substitution of the wheat flour by whole rice flour
}

\author{
Myriam de las Mercedes Salas Mellado ${ }^{1,2}$, Monika Haros ${ }^{1 *}$ \\ 1 Instituto de Agroquímica y Tecnología de Alimentos (IATA), Consejo Superior de Investigaciones Científicas (CSIC), Grupo Cereales, Paterna/Valencia - Spain \\ 2 Universidade Federal do Rio Grande (FURG), Escola de Química e Alimentos, Rio Grande/RS - Brasil
}

\section{${ }^{*}$ Corresponding Author:}

Monika Haros, Instituto de Agroquímica y Tecnología de Alimentos (IATA), Consejo Superior de Investigaciones Científicas (CSIC), Grupo Cereales, Av. Agustín Escardino, 7, Parque Científico, 46980, Paterna/Valencia - Spain, e-mail: mharos@iata.csic.es

Cite as: Evaluation of the technological, nutritional and sensory qualities of bakery products produced with partial substitution of the wheat flour by whole rice flour. Braz. J. Food Technol., v. 19, e2016002, 2016.

Received: Jan. 04, 2016; Accepted: July 27, 2016

\section{Resumen}

El objetivo del presente trabajo fue determinar las características tecnológicas, nutricionales y sensoriales del pan elaborado por sustitución de 25 y 50\% de la harina de trigo convencional por harina integral de arroz (Oryza sativa, L). Los productos desarrollados se evaluaron en términos de volumen específico del pan, aspecto de la rebanada central, dureza de la miga, contenido de humedad, fibra dietética, fitatos, minerales, cinética de retrogradación de la amilopectina por calorímetro diferencial de barrido y evaluación sensorial mediante escala hedónica de nueve puntos. En general, la incorporación de la harina integral de arroz a la formulación mostró diferencias significativas en cuanto a la cinética de retrogradación de la amilopectina en comparación con las muestras control (entalpía de retrogradación varió desde 2,4 a 1,8-2,1 J/g). También repercutió en una disminución del volumen de la pieza panaria (desde 4,48 a 2,26-2,86 cm³/g) que estuvo directamente relacionado con un aumento de la dureza de la miga (desde 0,77 a 1,90-3,85 N). No obstante, se observó una alta aceptación global por parte del consumidor (7,2-7,3). En cuanto a los minerales, el Ca y el Zn podrían estar biodisponibles en la formulación del $25 \%$ y en las muestras controles. En los productos con harina integral de arroz se observó un aumento significativo del nivel de trifosfatos de mio-inositol (desde valores despreciables a 0,38-0,44 $\mu \mathrm{moles} / \mathrm{g}$ ), el cual se considera que ejerce actividad biológica positiva en el organismo. Es importante considerar que la biodisponibilidad de minerales en los productos integrales está relacionada con la formulación y el proceso de elaboración del pan, principalmente por la presencia de fitatos. Éstos están presentes en alta proporción en la formulación con 50\% de harina integral de arroz, por lo que se estima una baja biodisponibilidad de minerales debido a que las relaciones molares fitato/mineral superan los valores de umbral de inhibición de la absorción de hierro y zinc.

Palabras clave: Harina integral de arroz; Calidad de pan; Biodisponibilidad de minerales; Fitatos; Evaluación sensorial; Retrogradación de la amilopectina.

\section{Summary}

The objective of this work was to determine the technological, nutritional and sensory characteristics of bread produced by substituting 25 and $50 \%$ of conventional wheat flour by whole rice flour (Oryza sativa $L$ ). The final products were evaluated in terms of specific loaf volume, appearance of the central slice, crumb firmness, moisture content, dietary fibre, phytates, minerals, kinetics of amylopectin retrogradation determined by differential scanning calorimetry, and a sensory evaluation using a 9-point hedonic scale. In general, the addition of whole rice flour to the formulation showed significant differences in the kinetics of amylopectin retrogradation as comparing to the control sample (retrogradation enthalpy changed from 2.4 to $1.8-2.1 \mathrm{~J} / \mathrm{g}$ ). It also caused a decrease in the specific volume of the bread (from 4.48 to $2.26-2.86 \mathrm{~cm}^{3} / \mathrm{g}$ ) which was directly related to an increase in crumb firmness (from 0.77 to 1.90-3.85 N). However, good overall sensory consumer 
Evaluación de la calidad tecnológica, nutricional y sensorial de productos de panadería por sustitución de harina de trigo por harina integral de arroz

Mellado, M. M. S.; Haros, M.

acceptance (7.2-7.3) was observed. As for the minerals, calcium and zinc may be bioavailable in the $25 \%$ formulation and in the control samples. In products with whole rice flour, a significant increase in the triphosphate myo-inositol level (from negligible values to 0.38-0.44 $\mu$ moles $/ \mathrm{g}$ ) was observed, which is considered to exert positive biological activity in the body. It is important to consider that mineral bioavailability in whole grain products is related to the formulation and the bread-making process, basically due to the presence of phytates. In fact, a high proportion of phytates was present in the formulation with $50 \%$ of whole rice flour, so low mineral bioavailability was expected as predicted by phytate/mineral molar ratios above the threshold for the inhibition values for iron and zinc absorption.

Keywords: Whole rice flour; Bread quality; Mineral availability; Phytates; Sensory evaluation; Amylopectin retrogradation.

\section{Introducción}

En los últimos años han surgido cambios relacionados con las costumbres alimenticias entre los cuales está el interés por los alimentos con cierto valor añadido como son los ricos en fibra dietética, ácidos grasos omega-3, probióticos, y prebióticos (ESPANHA, 2012). En consecuencia, investigaciones en materia de nutrición apuntan por otras alternativas al uso del trigo para la elaboración de pan.

Actualmente, el arroz (Oryza sativa L.) es un alimento básico en gran parte de la población mundial representando en torno al $50 \%$ de la producción de granos. Aproximadamente el $90 \%$ es producido y consumido en Asia, pero América Latina es la segunda en producción y la tercera en consumo de este cereal (RIO GRANDE DO SUL, 2011). Particularmente en Brasil el consumo de arroz es superior al de trigo debido a la limitada producción de éste, lo que conlleva al incremento de las importaciones de este cereal para la elaboración de productos de panadería (BRUM; HECK, 2005). Además, en poblaciones de regiones tropicales y sub-tropicales, el arroz es un cereal de alto rendimiento y bajo coste, siendo un cultivo prometedor para la preparación de productos de panadería (NAKAMURA et al., 2009). De hecho, el pan elaborado con harina de arroz integral supone una excelente fuente de energía y nutrientes (HEINEMANN et al., 2005), además de contener un amplio espectro de compuestos fenólicos, incluyendo derivados de los ácidos benzoico y cinámico, los cuales están dotados de múltiples actividades biológicas (CHUN et al. 2005). Desde el punto de vista tecnológico, debido a las distintas propiedades funcionales del almidón de arroz, entre otros usos, puede emplearse en la elaboración de pan por sustitución de harina de trigo. Además las proteínas presentes en el arroz, están compuestas mayoritariamente de albúminas y globulinas, y de una proporción insignificante de prolaminas, las cuales están implicadas en la formación del gluten. Por tanto, es posible su utilización en formulación de productos aptos para celíacos y a su vez revalorizar su cultivo para ser introducido en la alimentación de poblaciones con riesgo nutricional.

Por tanto, el objetivo del presente estudio fue desarrollar productos de panadería con mayor valor nutricional por sustitución de harina de trigo convencional por harina integral de arroz como alternativa a éste. Con este fin se investigó el efecto de la sustitución en la calidad de los productos desarrollados desde el punto de vista tecnológico, nutricional y sensorial.

\section{Material y métodos}

\subsection{Material}

En la elaboración de los productos de panadería, se utilizó harina refinada y harina integral de trigo de origen española y harina integral de arroz (Cerealle Indústria e Comercio de Cereais Ltda, Pelotas, Brasil). Para el desarrollo de la masa se empleó levadura prensada comercial (Saccharomyces cerevisiae, Levamax, España), agua potable y sal común.

\subsection{Métodos}

\subsubsection{Determinación de la absorción de agua}

Se evaluaron las propiedades de la masa mediante un Farinógrafo (Brabender, Duisburg, Alemania). Se utilizaron $300 \mathrm{~g}$ de cada formulación que fueron amasados en la amasadora del farinógrafo, termostatado a $30{ }^{\circ} \mathrm{C}$ hasta obtener 500 Unidades Brabender (UB).

\subsubsection{Formulación de la masa y proceso de panificación}

La formulación básica empleada en la elaboración de pan en base a $500 \mathrm{~g}$ de harina consistió en harina de trigo refinada e integral al 100\% (control) y mezclas por sustitución de harina refinada de trigo por $25 \%$ o 50\% de harina integral de arroz. A todas las formulaciones se les adicionó sal al 1,6\%, levadura prensada comercial al $5 \%$ y agua potable correspondiente a consistencia óptima según farinógrafo de Brabender (Tabla 1).

Para la elaboración de la masa se empleó el método esponja. Para ello se preparó una primera masa de $250 \mathrm{~g}$ de harina, a la que se le agregó el 50\% del agua total a añadir y $25 \mathrm{~g}$ de levadura. Seguidamente se dejó fermentar durante 24 horas a $4{ }^{\circ} \mathrm{C}$. Concluida esta etapa, se adicionaron el resto de los ingredientes y se mezclaron en amasadora (Mahot, Francia) hasta el desarrollo de la masa. Posteriormente, la masa se dejó reposar durante 10 minutos y se dividió en piezas de $100 \mathrm{~g}$ a las que se les efectuó un formado manual hasta 
Evaluación de la calidad tecnológica, nutricional y sensorial de productos de panadería por sustitución de harina de trigo por harina integral de arroz

Mellado, M. M. S.; Haros, M.

adquirir una forma esférica. Las piezas se fermentaron en una cámara de fermentación (Infrisa, España) a $28{ }^{\circ} \mathrm{C}$ y humedad relativa de $80 \%$ hasta el desarrollo óptimo del volumen. A continuación, las piezas se hornearon en horno eléctrico (Eurofours, 59144 Gommegnies, Francia) bajo condiciones controladas de tiempo y temperatura según formulación (Tabla 1). Finalmente, los panes fueron enfriados a temperatura ambiente durante una hora para su posterior análisis. Los experimentos fueron realizados por duplicado.

\subsubsection{Composición química y nutricional de las materias primas y productos desarrollados}

Se determinó la composición química de las materias primas en términos del contenido de humedad por gravimetría (método AACC 44-15a (AACC, 1995) cenizas por ignición a $910{ }^{\circ} \mathrm{C}$, contenido en lípidos por Soxhlet (método 960.39) (HORWITZ, 2000), contenido de proteínas por el método semimicro de Kjeldhal (método AACC 46-13) (AACC, 1995) y el contenido de fibra dietética soluble e insoluble por gravimetría (método 991.43) (AOAC, 1991). La concentración de fitatos y fosfatos de mio-inositol de menor grado de fosforilación fueron determinados por cromatografía líquida de alta resolución en fase reversa con detector de índice de refracción, siguiendo el método descrito por Sanz-Penella et al. (2008). Los ensayos se realizaron por duplicado. La caracterización de las materias primas utilizadas en el presente estudio se muestra en el Tabla 2.

Los productos de panadería se caracterizaron en términos de humedad, fibra dietética (soluble e insoluble) y fosfatos de mio-inositol, realizándose los ensayos por triplicado.

La determinación de minerales, Ca, Fe y Zn, consistió inicialmente en una etapa de digestión ácida de harina o pan en reactores de teflón empleando ácido nítrico concentrado y agua oxigenada al 30\% en proporciones 1:4, digiriéndose en un horno de microondas (Mars $X$, Cempor, USA). Se aplicó un ciclo de calentamiento de $180{ }^{\circ} \mathrm{C}$ durante 15 min y posterior enfriamiento durante el mismo tiempo. Seguidamente, la disolución obtenida se aforó a $10 \mathrm{ml}$ con $\mathrm{HCl}$ al $5 \%$ (v/v). Las muestras líquidas fueron analizadas por espectrometría de masas con fuente de plasma de acoplamiento inductivo, en el Servei Central de Suport a la Investigación Experimental de la Universidad de Valencia (Espanha). Los ensayos se realizaron por cuadruplicado.

\subsubsection{Parámetros tecnológicos de los productos desarrollados}

Se analizó el volumen específico de la pieza panaria $\left(\mathrm{cm}^{3} / \mathrm{g}\right)$, la relación de aspecto de la rebanada central $(\mathrm{cm} / \mathrm{cm})$ y dureza de la miga $(\mathrm{N})$, a partir del análisis del

Tabla 1. Parámetros de optimización para cada formulación estudiada.

\begin{tabular}{|c|c|c|c|c|c|c|c|}
\hline Muestra $^{a}$ & $\begin{array}{c}\text { Sustitución } \\
\text { de harina } \\
(\%)\end{array}$ & $\begin{array}{c}\text { Agua } \\
(\%)\end{array}$ & $\begin{array}{l}\text { Tiempo de } \\
\text { amasado } \\
\text { (min) }\end{array}$ & $\begin{array}{c}\text { Tiempo de } \\
\text { fermentación } \\
\text { (min) }\end{array}$ & $\begin{array}{l}\text { Volume } \\
\text { óptimo } \\
\text { (ml) }\end{array}$ & $\begin{array}{c}\text { Temperatura } \\
\text { de horneado } \\
\left({ }^{\circ} \mathrm{C}\right)\end{array}$ & $\begin{array}{c}\text { Tiempo de } \\
\text { horneado } \\
\text { (min) }\end{array}$ \\
\hline $\mathrm{HBC}$ & 0 & 60 & 5,3 & 25 & 110 & 180 & 20 \\
\hline $\mathrm{HIC}$ & 0 & 61 & 5,4 & 25 & 105 & 165 & 30 \\
\hline $25 \mathrm{HA}$ & 25 & 64 & 4,0 & 15 & 100 & 165 & 23 \\
\hline $50 \mathrm{HA}$ & 50 & 65 & 5,0 & 15 & 90 & 160 & 28 \\
\hline
\end{tabular}

aHBC: pan control elaborado con 100\% de harina refinada de trigo; HIC: pan control elaborado con 100\% de harina integral de trigo; $25 \mathrm{HA}$ y $50 \mathrm{HA}: 25 \%$ y $50 \%$ de sustitución de harina refinada de trigo por harina de arroz integral, respectivamente.

Tabla 2. Composición química de las materias primas empleadas en este estudio en la elaboración de pan.

\begin{tabular}{|c|c|c|c|c|}
\hline \multirow[b]{2}{*}{ Parámetro } & \multirow[b]{2}{*}{ Unidades $^{c}$} & \multicolumn{3}{|c|}{ Harinas } \\
\hline & & Trigo & $\begin{array}{c}\text { Trigo } \\
\text { Integral }\end{array}$ & $\begin{array}{c}\text { Arroz } \\
\text { Integral }\end{array}$ \\
\hline Humedad $^{a}$ & $(\%)$ & $14,5 \pm 0,01$ & $14,0 \pm 0,02$ & $10,9 \pm 0,01$ \\
\hline Proteínas ${ }^{a \star \star}$ & $(\%)$ & $13,51 \pm 0,10$ & $11,66 \pm 0,03$ & $8,14 \pm 0,06$ \\
\hline Cenizasa $^{a}$ & $(\%)$ & $0,63 \pm 0,01$ & $1,36 \pm 0,00$ & $1,45 \pm 0,00$ \\
\hline Lípidosa & $(\%)$ & $1,37 \pm 0,02$ & $1,68 \pm 0,02$ & $2,42 \pm 0,03$ \\
\hline Fitatos $^{a}$ & $(\mu \mathrm{moles} / \mathrm{g})$ & n.d. & $7,64 \pm 0,02$ & $10,56 \pm 0,03$ \\
\hline Fibra total ${ }^{a *}$ & $(\%)$ & $3,4 \pm 0,2$ & $8,3 \pm 0,1$ & $3,7 \pm 0,2$ \\
\hline $\mathrm{Ca}{ }^{b}$ & $(\mathrm{mg} / 100 \mathrm{~g})$ & $15,3 \pm 0,5$ & $35,0 \pm 1,0$ & $22,1 \pm 0,1$ \\
\hline $\mathrm{Fe}^{\mathrm{b}}$ & $(\mathrm{mg} / 100 \mathrm{~g})$ & $1,29 \pm 0,09$ & $2,87 \pm 0,39$ & $0,86 \pm 0,09$ \\
\hline $\mathrm{Zn}^{\mathrm{b}}$ & $(\mathrm{mg} / 100 \mathrm{~g})$ & $1,61 \pm 0,23$ & $3,31 \pm 0,09$ & $2,605 \pm 0,04$ \\
\hline
\end{tabular}

Media \pm DS; ${ }^{a} n=2 ;{ }^{b} n=4 ;$ ' Unidades expresadas en base seca. $n . d .=$ no detectado; *suma de fibra soluble mas fibra insoluble; ${ }^{*} N \times 5,7$. 
Evaluación de la calidad tecnológica, nutricional y sensorial de productos de panadería por sustitución de harina de trigo por harina integral de arroz

Mellado, M. M. S.; Haros, M.

perfil de textura de la miga por medio de un texturómetro TA-XT plus (Stable Micro System, Surrey, Reino Unido) (HAROS et al., 2001).

Las propiedades térmicas del almidón de la masa panaria durante el proceso de cocción y durante el almacenamiento del pan se analizaron por medio de un calorímetro diferencial de barrido (DSC7, Perkin Elmer). Inicialmente, el equipo fue calibrado con indio y zinc tomando como referencia una cápsula vacía de acero inoxidable (LVC 0319-0218, Perkin Elmer). Seguidamente las muestras de masa fueron pesadas en balanza de alta precisión, encapsuladas y selladas mediante prensado. Para simular el perfil de temperaturas en el centro de la miga de la pieza panaria durante su horneado, se emplearon las siguientes condiciones: 1. isoterma a $30{ }^{\circ} \mathrm{C}$ durante $1 \mathrm{~min} ; 2$. ciclo de calentamiento desde 30 a $100^{\circ} \mathrm{C}$ a una velocidad de $10^{\circ} \mathrm{C} / \mathrm{min}$; 3. isoterma a $100{ }^{\circ} \mathrm{C}$ durante 5 minutos; y 4. un ciclo de enfriamiento desde 100 hasta $30^{\circ} \mathrm{C}$ a una velocidad de $50{ }^{\circ} \mathrm{C} / \mathrm{min}$ (SANZ-PENELLA et al., 2010). Para estudiar la retrogradación de la amilopectina, las muestras se almacenaron a $4{ }^{\circ} \mathrm{C}$ durante 0, 1, 2, 3, 5, 7 y 14 días. Concluido cada intervalo de tiempo, a cada muestra se le aplicó un nuevo ciclo de calentamiento desde 30 a $130{ }^{\circ} \mathrm{C}$ a una velocidad de $10^{\circ} \mathrm{C} / \mathrm{min}$ (SANZ-PENELLA et al., 2010). Los parámetros evaluados fueron la temperatura inicial, temperatura de pico, temperatura final y entalpía de gelatinización y de retrogradación en $\mathrm{J} / \mathrm{g}$ de masa, representadas por el área bajo la curva del pico endotérmico correspondiente.

\subsubsection{Análisis sensorial}

El análisis sensorial se llevó a cabo con la participación de 50 catadores no entrenados. La aceptabilidad de cada producto desarrollado por los consumidores se evaluó mediante una escala hedónica de nueve puntos: 9. Me gusta muchísimo, 8. me gusta mucho, 7. me gusta moderadamente, 6. me gusta poco, 5. ni me gusta ni me disgusta, 4. me disgusta poco, 3. me disgusta moderadamente, 2. me disgusta mucho, 1. me disgusta muchísimo.

\subsubsection{Análisis estadístico}

Los resultados se analizaron estadísticamente por comparación múltiple en el programa Statgraphics Plus 7.1 por medio de la prueba de Fisher para establecer diferencias significativas mínimas entre muestras (LSD) a un nivel de significación de $5 \%(p<0,05)$.

\section{Resultados y discusión}

\subsection{Efecto de la inclusión de harina integral de arroz en las características tecnológicas de los productos desarrollados}

La humedad final de los productos desarrollados con harinas integrales mostró un escaso incremento, en algunos casos significativo, a pesar de la inclusión de salvado en las formulaciones. Esto se debe fundamentalmente a que en la formulación base se empleó harina de fuerza. Estas harinas presentan un alto contenido en gluten con una fuerza de $280 \times 10^{-4} \mathrm{~J}$, según datos alveográficos obtenidos en el laboratorio. La inclusión de las harinas integrales de trigo y de arroz en las formulaciones provocó una disminución significativa en cuanto al volumen de la pieza panaria en función del porcentaje de sustitución (Tabla 3). Así pues, el menor volumen y mayor dureza de la miga, lo presentaron las formulaciones con $50 \%$ de incorporación de harina de arroz (Figura 1). Al tratarse de harinas integrales la presencia de salvado interfirió en la formación de la red de

Tabla 3. Efecto de la formulación de pan en la composición y calidad de los panes desarrollados.

\begin{tabular}{|c|c|c|c|c|c|}
\hline \multirow[t]{2}{*}{ Parámetro } & \multirow[t]{2}{*}{ Unidades } & \multicolumn{2}{|c|}{$\begin{array}{l}\text { Harina de Trigo } \\
\qquad 100 \%\end{array}$} & \multicolumn{2}{|c|}{$\begin{array}{c}\text { Harina Integral de } \\
\text { Arroz }\end{array}$} \\
\hline & & Refinada & Integral & $25 \%$ & $\mathbf{5 0} \%$ \\
\hline Humedad $^{1}$ & $\%$ & $33,4 \pm 0,3^{a}$ & $33,4 \pm 0,9^{a}$ & $34,1 \pm 0,4^{a}$ & $35,6^{b}$ \\
\hline Vol. Específico² & $\mathrm{ml} / \mathrm{g}$ & $4,48 \pm 0,46^{d}$ & $3,83 \pm 0,24^{c}$ & $2,86 \pm 0,22^{b}$ & $2,26 \pm 0,02^{a}$ \\
\hline Ancho/alto & $\mathrm{cm} / \mathrm{cm}$ & $1,65 \pm 0,14^{b}$ & $1,66 \pm 0,14^{b}$ & $1,54 \pm 0,03^{a}$ & $1,71 \pm 0,05^{b}$ \\
\hline Dureza² $^{2}$ & $\mathrm{~N}$ & $0,77 \pm 0,09^{a}$ & $1,03 \pm 0,05^{b}$ & $1,90 \pm 0,14^{c}$ & $3,85 \pm 0,13^{d}$ \\
\hline Aceptabilidad ${ }^{3}$ & & $7,9^{\mathrm{b}}$ & $8,2^{c}$ & $7,3^{\mathrm{ab}}$ & $7,2^{\mathrm{a}}$ \\
\hline Fibra Dietética Insoluble ${ }^{1}$ & $\%$, b.s. & $3,1 \pm 0,4^{a}$ & $8,3 \pm 0,5^{b}$ & $3,2 \pm 0,3^{a}$ & $3,3 \pm 0,0^{\mathrm{a}}$ \\
\hline Fibra Dietética Soluble ${ }^{1}$ & $\%$, b.s. & $2,8 \pm 0,5^{b}$ & $3,0 \pm 0,1^{b}$ & $2,5 \pm 0,2^{b}$ & $2,1 \pm 0,1^{b}$ \\
\hline Fibra Dietética Total ${ }^{1}$ & $\%$, b.s. & $5,9 \pm 0,1^{\mathrm{a}}$ & $11,3 \pm 0,4^{b}$ & $5,7 \pm 0,1^{a}$ & $5,4 \pm 0,1^{a}$ \\
\hline $\operatorname{lns} P_{6}^{1}$ & $\mu \mathrm{mol} / \mathrm{g}, \mathrm{b} . \mathrm{s}$ & n.d & $2,6 \pm 0,1^{b}$ & $1,6 \pm 0,1^{a}$ & $3,8 \pm 0,3^{c}$ \\
\hline $\operatorname{lns} P_{5}^{1}$ & $\mu \mathrm{mol} / \mathrm{g}$, b.s & n.d & $0,63 \pm 0,01^{b}$ & $0,38 \pm 0,05^{a}$ & $0,74 \pm 0,04^{\circ}$ \\
\hline $\operatorname{lns} P_{4}^{1}$ & $\mu \mathrm{mol} / \mathrm{g}, \mathrm{b} . \mathrm{s}$ & n.d & $0,30 \pm 0,01^{a}$ & $0,27 \pm 0,04^{a}$ & $0,29 \pm 0,05^{a}$ \\
\hline $\operatorname{lns} P_{3}^{1}$ & $\mu \mathrm{mol} / \mathrm{g}$, b.s & n.d & $0,50 \pm 0,00^{c}$ & $0,44 \pm 0,02^{b}$ & $0,38 \pm 0,03^{a}$ \\
\hline $\operatorname{lns} P_{6}+\operatorname{Ins} P_{5}^{1}$ & $\mu \mathrm{mol} / \mathrm{g}$, b.s & n.d & $3,2 \pm 0,1^{b}$ & $2,0 \pm 0,1^{\mathrm{a}}$ & $4,6 \pm 0,3^{c}$ \\
\hline
\end{tabular}

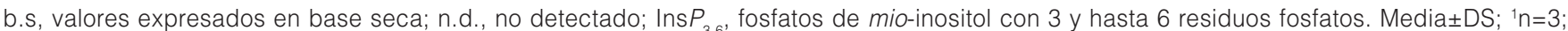
${ }^{2} n=6 ;{ }^{3} n=50$. Valores seguidos de la misma letra en la misma línea no son significativamente diferentes a un nivel de confianza de $95 \%$. 
Evaluación de la calidad tecnológica, nutricional y sensorial de productos de panadería por sustitución de harina de trigo por harina integral de arroz

Mellado, M. M. S.; Haros, M.

HBC
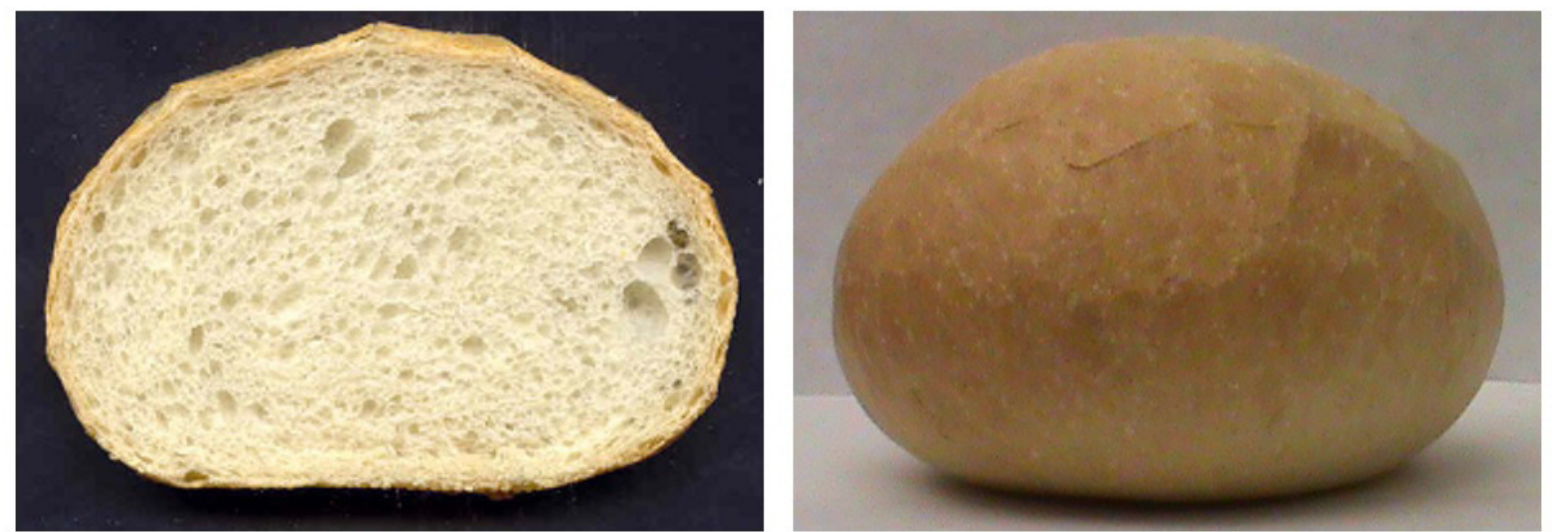

HIC
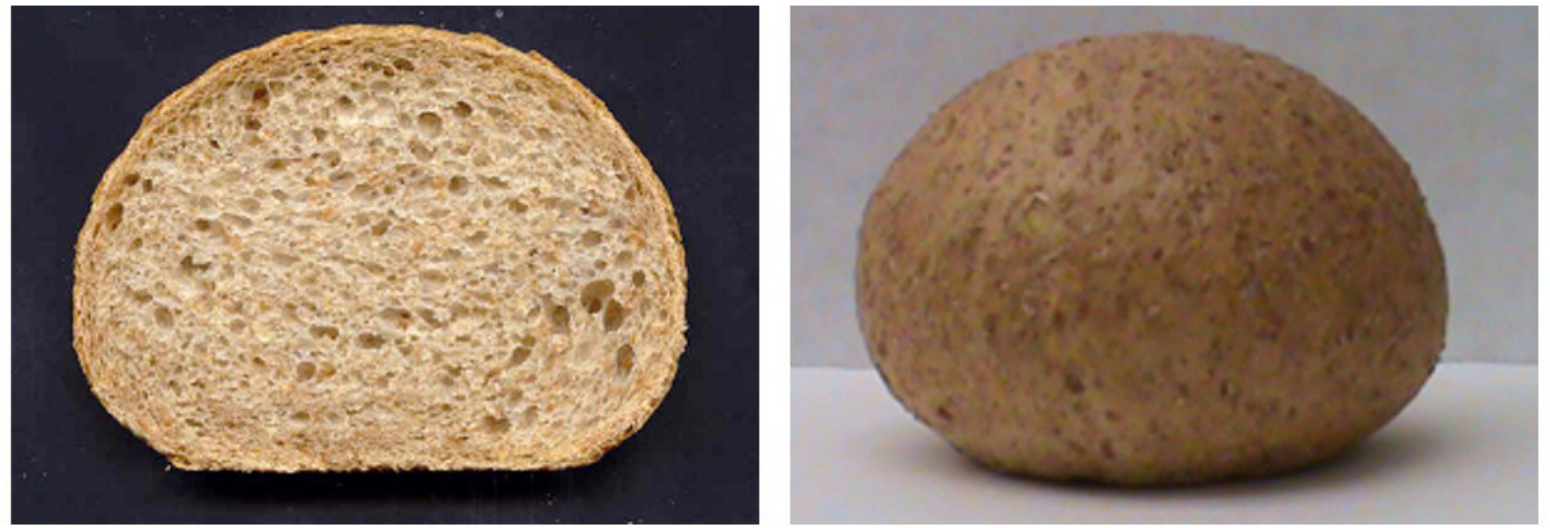

25HA
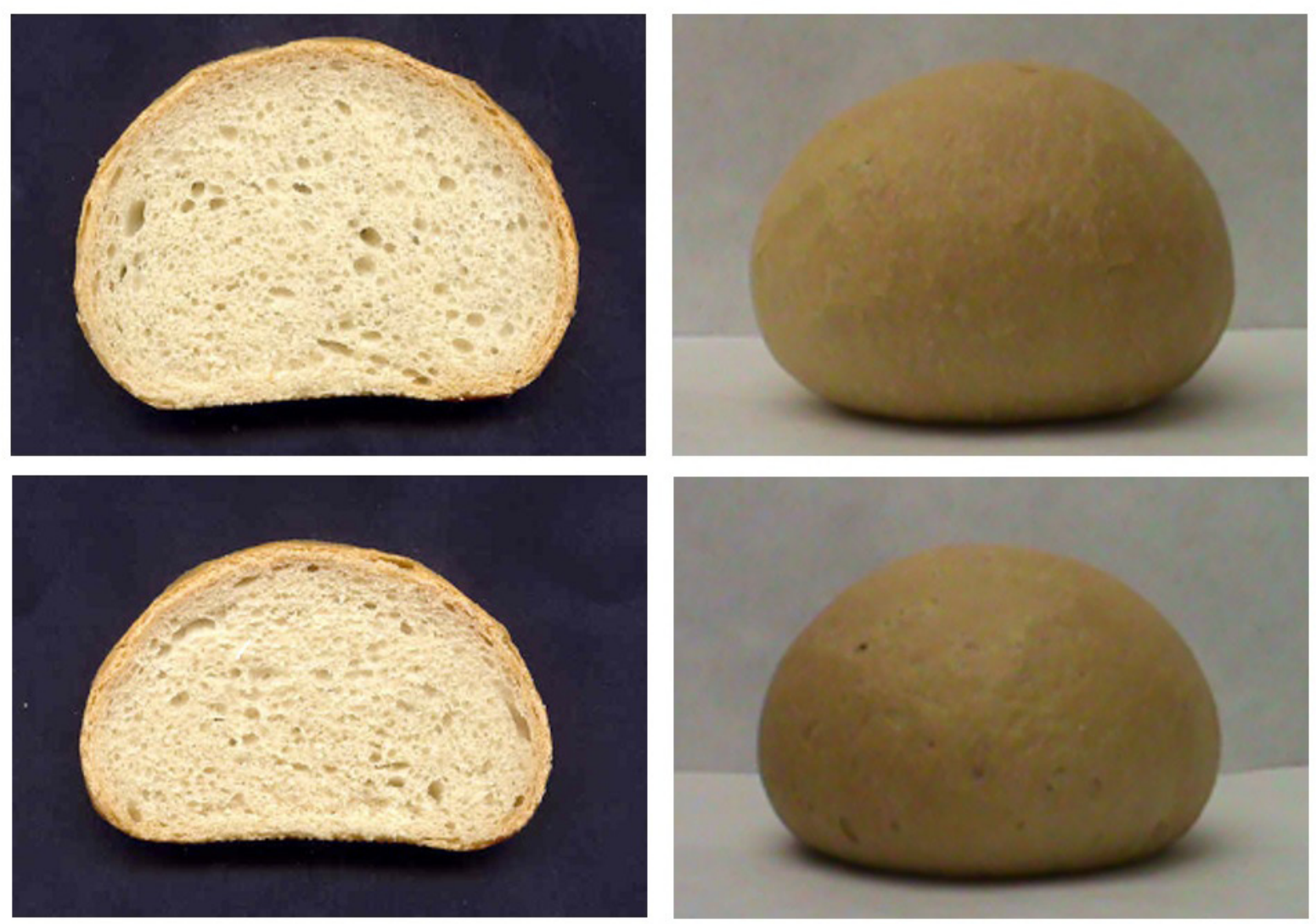

50HA

Figura 1. Efecto de la incorporación de harina integral de arroz en el volumen y la relación de aspecto de la rebanada central. HBC: pan control elaborado con 100\% de harina refinada de trigo; HIC: pan control elaborado con 100\% de harina integral de trigo; $25 \mathrm{HA}$ y $50 \mathrm{HA}: 25 \%$ y $50 \%$ de sustitución de harina refinada de trigo por harina de arroz integral, respectivamente. 
Evaluación de la calidad tecnológica, nutricional y sensorial de productos de panadería por sustitución de harina de trigo por harina integral de arroz

Mellado, M. M. S.; Haros, M.

gluten afectando al desarrollo del volumen y a la estructura de la miga. Esto impidió la expansión de la masa durante la fermentación, debido a la interferencia del salvado en la eficiente formación y desarrollo de la estructura de los alveolos (SANZ-PENELLA et al., 2010). Particularmente, para el caso del arroz este efecto se debió fundamentalmente a que las harinas de arroz carecen de gluten, por lo tanto sus características viscoelásticas son muy diferentes al trigo así como una baja proporción, incluso insignificante, de proteínas implicadas en la formación del gluten (DEL CASTILLO et al., 2009; ALVIS et al., 2011). Sin embargo, la relación de aspecto de la rebanada central no se vio afectada (Tabla 3). La adición del $25 \%$ de harina de arroz incluso mejoró ligeramente la relación de aspecto en comparación con los controles de trigo (Figura 1).

\subsection{Evaluación sensorial y aceptación por parte del consumidor}

La Tabla 3 muestra la puntuación media obtenida para cada formulación por parte de los consumidores. Los resultados verifican que la mayor aceptación corresponde a los panes control de trigo situándose en el nivel correspondiente a 8 puntos ("me gusta mucho").

En un valor medio de 7 puntos dentro de la escala hedónica y por tanto en un grado inferior de aceptabilidad ("me gusta moderadamente"), se situaron los panes adicionados con harina de arroz. Estos resultados indican que existe una pérdida de calidad sensorial ocasionada por la inclusión de esta harina. El principal defecto detectado por el consumidor en los panes con un 50\% de arroz fue la textura definida como "densa" o "compacta".

\subsection{Efecto de la inclusión de las distintas materias primas en la composición química de los productos desarrollados}

En la Tabla 3 también se recogen los valores obtenidos en cuanto a la composición química de los panes elaborados. Debido a las diferencias encontradas en cuanto a la composición química de las distintas materias primas, el producto final, se vio influenciado en el mismo sentido. Los productos a los que se les incorporó harina integral de arroz presentaron un contenido en fibra total significativamente inferior al pan control elaborado con harina integral de trigo. Sin embargo, en relación al contenido en fibra dietética soluble, estos panes no presentaron diferencias significativas al pan elaborado con harina integral de trigo, por lo tanto son equivalentes en cuanto al contenido de este componente. En cuanto al efecto de la formulación en el contenido de fitatos residual y de fosfatos de mio-inositol de menor grado de fosforilación generados durante la fermentación del pan, los resultados también se muestran en la Tabla 2. Como cabría esperar no se han detectado fosfatos de mio-inositol en la formulación de pan blanco. El contenido de fitatos disminuyó drásticamente respecto al valor inicial y teniendo en cuenta las proporciones de cada una de ellas en las formulaciones de pan integral y adicionadas con harina integral de arroz. Esto se debió fundamentalmente a la actividad fitasa endógena de las propias harinas, las cuales se activan durante las etapas de amasado, reposo, fermentación y primera etapa de cocción (LOPEZ et al., 2001). Teniendo en cuenta que el pentakisfosfato de mio-inositol $\left(\operatorname{lns} P_{5}\right)$ también causa efectos inhibitorios en la biodisponibilidad mineral se observó que el mayor contenido de $\operatorname{lns} P_{6}+\operatorname{lns} P_{5}$ lo presentó el pan elaborado con $50 \%$ de harina integral de arroz, mostrando valores significativamente superiores al resto de formulaciones. Por otro lado, el contenido de trisfosfato de mio-inositol $\left(\operatorname{lns} P_{3}\right)$, el cual está implicado en numerosas funciones biológicas en nuestro organismo, fue estadísticamente mayor en el pan integral, seguido del pan elaborado con solo $25 \%$ de harina integral de arroz (Tabla 3). Desde el punto de vista nutricional y funcional, los fosfatos de mio-inositol de menor grado de fosforilación, como es el caso del Ins $P_{3}$, afectan en menor medida o no afectan la biodisponibilidad de minerales, sino que desempeñan diversas acciones beneficiosas en el organismo dotadas de efectos farmacológicos tales como antiinflamatorios, prevención de complicaciones diabéticas, implicación en el crecimiento y diferenciación celular, así como la regulación del calcio intracelular, entre otras funciones (SHI et al., 2006). Por tanto, es más recomendable consumir productos que presentan una hidrólisis avanzada de fitatos con acumulación de Ins $P_{3}$ (SANZ-PENELLA et al., 2009; HAROS et al., 2009) Estos resultados muestran que el pan con $25 \%$ de harina de arroz, presentó una mayor acumulación de $\operatorname{lns} P_{3}$, sugiriendo una mayor hidrólisis de Ins $P_{6}$ durante el proceso.

\subsection{Efecto de la formulación en el aporte de $\mathrm{Fe}, \mathrm{Ca}$ y Zn en la contribución a la ingesta diaria recomendada y predicción de la biodisponibilidad mineral}

La Tabla 4 muestra el efecto de la formulación en el aporte de calcio, hierro y zinc, así como los porcentajes teóricos de la contribución de cada mineral en base a una ingesta diaria de $100 \mathrm{~g}$ de pan acorde con las recomendaciones de la National Academy of Science (NAS, 2014). Los productos formulados con harina de arroz presentaron contenidos en Fe, Zn y Ca estadísticamente inferiores a los panes con harina integral de trigo. A pesar de que la harina de arroz integral presenta mayores contenidos de calcio y zinc que la harina blanca, en las formulaciones en las que fue adicionada no se observó la misma tendencia. Por otro lado se observó un descenso en el contenido de hierro (LAMBERTS et al., 2007).

Resulta importante conocer el aporte a la ingesta diaria recomendada de estos productos. Pero, aún es más 
Evaluación de la calidad tecnológica, nutricional y sensorial de productos de panadería por sustitución de harina de trigo por harina integral de arroz

Mellado, M. M. S.; Haros, M.

Tabla 4. Efecto de la formulación en el contenido de minerales, su contribución a la ingesta diaria recomendada y predicción de la biodisponibilidad mineral'1.

\begin{tabular}{|c|c|c|c|c|c|c|c|c|c|c|c|}
\hline \multirow{3}{*}{ Muestra } & \multicolumn{3}{|c|}{$\begin{array}{c}\text { Contenido en } \text { Minerales }^{2} \\
\text { mg/100 g pan b.s }\end{array}$} & \multicolumn{3}{|c|}{$\begin{array}{c}\text { Ratio Molar } \\
\text { Fitato/Mineral }\end{array}$} & \multicolumn{5}{|c|}{$\begin{array}{l}\text { Ingesta Diaria Recomendada } \\
\% \text { contribución/100 g pan }{ }^{* *}\end{array}$} \\
\hline & \multirow{2}{*}{$\mathrm{Fe}$} & \multirow{2}{*}{$\mathrm{Ca}$} & \multirow{2}{*}{ Zn } & \multirow{2}{*}{ Ins $P_{6} / F e$} & \multirow{2}{*}{ Ins $P_{6} / \mathrm{Ca}$} & \multirow{2}{*}{ Ins $P_{6} / Z n$} & \multicolumn{2}{|c|}{$\mathrm{Fe}$} & \multirow{2}{*}{$\frac{\mathrm{Ca}}{\text { Adulto }}$} & \multicolumn{2}{|c|}{$\mathrm{Zn}$} \\
\hline & & & & & & & $\mathrm{H}$ & M & & $\mathrm{H}$ & M \\
\hline $\mathrm{HBC}$ & $1,7 \pm 0,1^{b}$ & $70,0 \pm 0,8^{b}$ & $2,5 \pm 0,8^{a}$ & - & - & - & 19,48 & 8,64 & 7,56 & 20,68 & 29,36 \\
\hline $\mathrm{HIC}$ & $3,5 \pm 0,2^{c}$ & $80,8 \pm 0,2^{b}$ & $3,9 \pm 0,6^{a}$ & 4,11 & 0,12 & 4,71 & 40,60 & 18,04 & 7,84 & 32,76 & 45,04 \\
\hline $25 \mathrm{HA}$ & $1,2 \pm 0,1^{a}$ & $54,9 \pm 0,7^{b}$ & $3.0 \pm 0,4^{a}$ & 7,39 & 0,13 & 4,38 & 13,84 & 6,16 & 5,12 & 24,36 & 33,48 \\
\hline $50 \mathrm{HA}$ & $1,0 \pm 0,1^{a}$ & $60,1 \pm 0,8^{b}$ & $2,5 \pm 0,2^{a}$ & 19,76 & 0,24 & 9,39 & 11,40 & 5,04 & 5,48 & 22,60 & 31,08 \\
\hline
\end{tabular}

${ }^{1} \mathrm{HBC}$ : pan control elaborado con $100 \%$ de harina de trigo; HIC: pan control elaborado con $100 \%$ de harina integral de trigo; $25 \mathrm{HA}$ y $50 \mathrm{HA}$ $25 \%$ y $50 \%$ de sustitución de harina de trigo por harina de arroz integral, respectivamente; b.s: expresados en base seca; H: hombre, M: mujer; ${ }^{2}$ Media \pm DS; $n=4$; valores seguidos de la misma letra en la misma columna no son significativamente diferentes a un nivel de confianza de 95\%, **Ingesta diaria recomendada, contribución (\%) para uma ingesta de $100 \mathrm{~g}$ de pan. Ingesta diaria em mg/dia para adultos en Calcio es 1000 , para Hierro es $8 / 18$ hombre/mujer y para Zinc es 11/8 hombre/mujer, respectivamente. (NAS, 2014)

relevante predecir si su biodisponibilidad se encontrará comprometida o no por sustancias inhibitorias de la absorción de minerales. Asimismo, el grado en que estos minerales pueden ser absorbidos por el organismo está íntimamente ligado a la presencia de fitatos, debido a su poder quelante.

Por tanto, se han establecido valores umbral de las relaciones molares entre el fitatos y los minerales a partir de los cuales comenzaría la inhibición de la absorción de los mismos en el tracto gastrointestinal. Para el caso del hierro su valor umbral correspondería a un ratio molar de 1,0 , en cuanto al calcio, el umbral de biodisponibilidad es 0,24 y para el zinc hasta ratios molares de 5 (MA et al., 2005; HURRELL, 2004). En el caso del hierro, se observó que especialmente las formulaciones con harina integral de arroz cubrirían el 11,4 y 13,84\% de los requerimientos de este mineral en hombres, y entre un 5,04 y $6,16 \%$ de los requerimientos para las mujeres del total de la ingesta diaria recomendada en base a $100 \mathrm{~g}$ de pan, respectivamente. En particular, el pan elaborado con $100 \%$ de harina integral presentó el mayor porcentaje de contribución. Sin embargo, este mineral no se encontraría disponible en su totalidad en ninguno de los casos, de acuerdo a que los ratios superan el valor umbral de inhibición establecido. Por tanto, sería más recomendable el consumo de productos de panadería fortificados con fierro y elaborados con fermentaciones prolongadas para disminuir el contenido de fitatos para que afecte en menor proporción la biodisponibilidad de hierro (Tabla 4).

En el caso del calcio, éste se encontraría biodisponible en todas las formulaciones estudiadas. El zinc se presentaría biodisponible en todas las formulaciones ensayadas, con la excepción de la formulación con 50\% de harina integral de arroz. En general, los productos desarrollados destacan por alta proporción de este mineral contribuyendo a la ingesta diaria recomendada en más del 20\% en hombres y más de $30 \%$ en mujeres (Tabla 4).

\subsection{Propiedades térmicas del almidón durante la cocción y almacenamiento de las piezas panarias}

Las muestras adicionadas con harina integral de arroz no mostraron diferencias significativas entre las formulaciones ensayadas y en el producto elaborado con harina de trigo refinada (control) en cuanto a la temperatura y la entalpía de gelatinización, con un ligero incremento en la temperatura de pico en la muestra con 50\% de sustitución con arroz o harina integral. En el segundo ciclo de calentamiento tras el almacenamiento en refrigeración, se observaron dos picos, el primero de ellos corresponde al pico de retrogradación de la amilopectina, mientras que el segundo al de la fusión del complejo lípido-amilosa. Tras el almacenamiento se registró la evolución de la entalpía de retrogradación de la amilopectina, debido a que es uno de los principales mecanismos involucrados en el envejecimiento del pan. La entalpía de retrogradación se incrementó gradualmente con el tiempo de almacenamiento en todas las formulaciones ensayadas (resultados no mostrados). En la Tabla 5 se muestra el efecto de la formulación sobre la gelatinización del almidón durante el horneado simulado en el calorímetro diferencial de barrido. Al cabo de dos semanas la muestra control de harina de trigo refinada, presentó los valores más elevados de entalpía de retrogradación, con valores cercanos a $3 \mathrm{~J} / \mathrm{g}$ de masa. Esto indicaría un mayor endurecimiento de la miga de este producto con el almacenamiento. En general, las formulaciones adicionadas con harinas integrales mostraron menores valores de entalpías de retrogradación. Especialmente la formulación con 50\% de harina de arroz arrojó valores asintóticos de 2,5 J/g 
Evaluación de la calidad tecnológica, nutricional y sensorial de productos de panadería por sustitución de harina de trigo por harina integral de arroz

Mellado, M. M. S.; Haros, M.

Tabla 5. Efecto de la formulación en las propiedades térmicas del almidón.

\begin{tabular}{|c|c|c|c|c|c|}
\hline \multirow[t]{2}{*}{ Parámetro* } & \multirow[t]{2}{*}{ Unidades } & \multicolumn{2}{|c|}{$\begin{array}{c}\text { Harina de Trigo } \\
100 \%\end{array}$} & \multicolumn{2}{|c|}{$\begin{array}{c}\text { Harina Integral de } \\
\text { Arroz }\end{array}$} \\
\hline & & Refinada & Integral & $25 \%$ & $50 \%$ \\
\hline \multicolumn{6}{|c|}{ Gelatinización del almidón** } \\
\hline$T_{\text {inicio-G }}$ & ${ }^{\circ} \mathrm{C}$ & $65,8 \pm 0,7^{a}$ & $66,8 \pm 0,6^{b c}$ & $66,9 \pm 0,3^{c}$ & $66,4 \pm 0,6^{a b c}$ \\
\hline$T_{\text {pico-G }}$ & ${ }^{\circ} \mathrm{C}$ & $74,3 \pm 0,5^{a}$ & $75,2 \pm 0,5^{b}$ & $74,3 \pm 0,5^{\mathrm{a}}$ & $75,3 \pm 0,3^{b}$ \\
\hline$T_{\text {final-G }}$ & ${ }^{\circ} \mathrm{C}$ & $98,7 \pm 0,5^{b}$ & $91,5 \pm 0,6^{a}$ & $97,7 \pm 0,3^{b}$ & $98,1 \pm 0,7^{b}$ \\
\hline$\Delta \mathrm{H}_{\mathrm{G}}$ & $\mathrm{J} / \mathrm{g}$ b.s & $3,2 \pm 0,3^{b}$ & $3,3 \pm 0,2^{b}$ & $2,9 \pm 0,3^{a}$ & $3,4 \pm 0,1^{b}$ \\
\hline \multicolumn{6}{|c|}{ Retrogradación de la Amilopectina (3 días de almacenamiento) ${ }^{\star \star \star}$} \\
\hline $\mathrm{T}_{\text {inicio-R }}$ & ${ }^{\circ} \mathrm{C}$ & $42,1 \pm 0,5^{\mathrm{ab}}$ & $41,4 \pm 0,1^{a}$ & $41,2 \pm 0,7^{a}$ & $43,0 \pm 0,3^{b}$ \\
\hline $\mathrm{T}_{\text {pico-R }}$ & ${ }^{\circ} \mathrm{C}$ & $57,9 \pm 0,4^{a}$ & $57,2 \pm 0,1^{a}$ & $57,5 \pm 0,1^{a}$ & $57,9 \pm 0,5^{a}$ \\
\hline$T_{\text {final- } R}$ & ${ }^{\circ} \mathrm{C}$ & $73,0 \pm 0,6^{a}$ & $72,3 \pm 0,8^{a}$ & $71,7 \pm 0,3^{a}$ & $71,5 \pm 0,2^{a}$ \\
\hline$\Delta \mathrm{H}_{\mathrm{R}}$ & $\mathrm{J} / \mathrm{g}$ b.s & $2,4 \pm 0,1^{c}$ & $2,1 \pm 0,1^{\mathrm{ab}}$ & $2,1 \pm 0,1^{b}$ & $1,8 \pm 0,17^{a}$ \\
\hline
\end{tabular}

' $T_{\text {nn }}, T_{\text {rco }}$ y $T_{\text {final, }}$ temperaturas de inicio, de pico y final del pico endotérmico de gelatinización (G) o retrogradación (R); $\Delta H_{G}$ : Entalpía de gelatinización; $\Delta \mathrm{H}_{\mathrm{B}}$ : Entalpía de retrogradación de la amilopectina; b.s: base seca. Media $\pm \mathrm{DS} ;{ }^{* *} \mathrm{n}=12 ;{ }^{* * *} \mathrm{n}=3$; Valores seguidos de la misma letra en la misma fila no son significativamente diferentes a un nivel de confianza de $95 \%$.

de masa. Esto indicaría que esta formulación presentaría mayor vida útil comparado con los panes control de trigo. No obstante, profundizando en los resultados al cabo de 3 días de almacenamiento (Tabla 5), el pico endotérmico de retrogradación de las muestras de trigo, se encuentran entre $42,4-73,3^{\circ} \mathrm{C}$ y $41,9-72,8^{\circ} \mathrm{C}$ siendo su entalpía de retrogradación de 2,4 y 2,1 J/g de masa en la muestra con harina refinada y harina integral, respectivamente. Se observa, que el pan adicionado con 50\% de harina integral de arroz presenta entalpía de retrogradación de la amilopectina significativamente menor (1,8 J/g de masa) al resto de formulaciones. La alta proporción de aceite presente en la harina de arroz pudo interferir en la recristalización de la amilopectina, lo que favoreció a una disminución significativa de la velocidad de envejecimiento del pan (MONDRAGÓN et al., 2006). La presencia de fibras en la formulación también produce alteración del balance de agua entre los distintos componentes de la miga, favoreciendo en mayor o menor medida la velocidad y la magnitud del fenómeno de la retrogradación de la amilopectina.

\section{Conclusiones}

La sustitución de 25\% de harina integral de arroz no produjo grandes cambios en cuanto a los parámetros de calidad tecnológica, mostrando algunas características, como la relación de forma, superior al pan control. El pan con $25 \%$ de harina de arroz, presentó una mayor acumulación de $\operatorname{lns} P_{3}$, sugiriendo una mayor hidrólisis de Ins $P_{6}$ durante el proceso.

En todas las formulaciones estudiadas se predice una alta biodisponibilidad en cuanto al calcio y zinc, con la excepción de este último mineral en el producto con $50 \%$ de arroz. La adición de harina integral de arroz a la formulación provocó una disminución en la entalpía de retrogradación de la amilopectina, lo que podría incrementar la vida útil del producto en cuanto al envejecimiento de éste durante el almacenamiento. Las formulaciones estudiadas no supusieron un deterioro pronunciado de la calidad sensorial del producto final, con un alto grado de aceptabilidad por parte del consumidor, por lo que la harina integral de arroz podría ser un ingrediente idóneo para ser empleado en productos de panadería en alta proporción con implicación positiva en cuanto a la calidad tecnológica y nutricional del producto final.

\section{Agradecimientos}

Este trabajo fue financiado por los proyectos Consolider Fun-C-Food CSD2007-00063 del Ministerio de Economía y Competitividad (MINECO) y PROMETEO/2012/064 de la Generalitat Valenciana, España. Los autores desean agradecer a las investigadoras Dra. Dinoraz Velez y Dr. Vicenta Devesa del Grupo de Elementos Traza (IATA-CSIC) por su ayuda en la digestión de las muestras para la determinación de minerales y a la Ing. Marta Sancho-Robles por su excelente participación en esta investigación.

\section{Referencias}

ALVIS, A.; PEREZ, L. J.; ARRAZOLA, G. Estudio de propiedades físicas y viscoelásticas de panes elaborados con mezclas de harina de trigo y arroz integral. Información Tecnológica, La Serena, v. 22, n. 4, p. 107-115, 2011. http://dx.doi.org/10.4067/ S0718-07642011000400012.

AMERICAN ASSOCIATION OF CEREAL CHEMISTS - AACC. Approved methods of the American Association of Cereal Chemists. 9th ed. St Paul: AACC, 1995. 
Evaluación de la calidad tecnológica, nutricional y sensorial de productos de panadería por sustitución de harina de trigo por harina integral de arroz

Mellado, M. M. S.; Haros, M.

ASSOCIATION OF OFFICIAL ANALYTICAL CHEMISTS - AOAC. Official Methods of Analysis of the Association of Official Analytical Chemists. Arlington: AOAC, 1991.

BRUM, A. L.; HECK, C. R. A economia do trigo no Rio Grande do Sul. Breve histórico do cereal na economía do estado. Análise, Porto Alegre, v. 16, n. 1, p. 29-44, 2005

CHUN, O. K.; KIM, D. O.; SMITH, N.; SCHROEDER, D.; HAN, J T.; LEE, C. Y. Daily consumption of phenolics and total antioxidant capacity from fruit and vegetables in the American diet. Journal of the Science of Food and Agriculture, London, v. 85, n. 10, p. 1715-1724, 2005. http://dx.doi.org/10.1002/jsfa.2176.

DEL CASTILLO, V.; LESCANO, G.; ARMADA, M. Formulación de alimentos para celíacos con base en mezclas de harinas de quínoa, cereales y almidones. Archivos Latinoamericanos de Nutrición, Caracas, v. 59, n. 3, p. 332-336, 2009.

ESPANHA. Ministerio de Sanidad y Consumo. Agencia Española de Seguridade Alimentaria. Estrategia NAOS: estrategia para la nutrición, actividad física y prevención de la obesidad. Madrid, 2012. Disponible: <http://www.naos.aesan.msssi.gob.es/naos/ estrategia/que-es/>. Acceso: 20 oct. 2015.

HAROS, M.; CARLSSON, N.; ALMGREN, A.; LARSSON-ALMINGER, M.; SANDBERG, A. S.; ANDLID, T. Phytate degradation by human gut isolated Bifidobacterium pseudocatenulatum ATCC 27919 and its probiotic potential. International Journal of Food Microbiology, Amsterdam, v. 135, n. 1, p. 7-14, 2009. http:// dx.doi.org/10.1016/j.ijfoodmicro.2009.07.015. PMid:19674804.

HAROS, M.; ROSELL, C. M.; BENEDITO, C. Use of fungal phytase to improve breadmaking performance of whole wheat bread. Journal of Agricultural and Food Chemistry, Washington, v. 49, n. 11, p. 5450-5454, 2001. http://dx.doi.org/10.1021/jf010642l. PMid:11714342

HEINEMANN, R. J. B.; FAGUNDES, P. L.; PINTO, E. A.; PENTEADO, M. V. C.; LANFER-MARQUEZ, U. M. Comparative study of nutrient composition of commercial brown, parboiled and milled rice from Brazil. Journal of Food Composition and Analysis, San Diego, v. 18, n. 4, p. 287-296, 2005. http://dx.doi.org/10.1016/j. jfca.2004.07.005.

HORWITZ, W. (Ed.). Official methods of analysis of the Association of Official Analytical Chemists. Gaithersburg: AOAC, 2000

HURRELL, R. F. Phytic acid degradation as a means of improving iron absorption. International Journal for Vitamin and Nutrition Research, Bern, v. 74, n. 6, p. 445-452, 2004. http://dx.doi. org/10.1024/0300-9831.74.6.445. PMid:15743020.

LAMBERTS, L.; DEBIE, E.; VANDEPUTTE, E. G.; VERAVERBEKE, W. S.; DERYCKE, V.; DE MAN, W.; DELCOUR, J. A. Effect of milling on colour and nutritional properties of rice. Food
Chemistry, London, v. 100, n. 4, p. 1496-1501, 2007. http:// dx.doi.org/10.1016/j.foodchem.2005.11.042

LOPEZ, H. W.; KRESPINE, V.; GUY, C.; MESSAGER, A.; DEMIGNE, C.; REMESY, C. Prolonged fermentation of whole wheat sourdough reduces phytate level and increases soluble magnesium. Journal of Agricultural and Food Chemistry, Washington, v. 49, n. 5, p. 2657-2662, 2001. http://dx.doi.org/10.1021/jf001255z. PMid:11368651

MA, G.; JIN, Y.; PLAO, J.; KOK, F.; JACOBSEN, E. Phytate, calcium, iron, and zinc contents and their molar ratios in foods commonly consumed in China. Journal of Agricultural and Food Chemistry, Washington, v. 53, n. 26, p. 10285-10290, 2005. http://dx.doi.org/10.1021/jf052051r. PMid:16366728.

MONDRAGÓN, M.; MENDOZA-MARTÍNEZ, A. M.; BELLO-PÉREZ, L. A.; PEÑA, J. L. Viscoelastic behavior of nixtamalized maize starch gels. Carbohydrate Polymers, Barking, v. 65, n. 3, p. 314-320, 2006. http://dx.doi.org/10.1016/j.carbpol.2006.01.011.

NAKAMURA, S.; SUZUKI, K.; OHTSUBO, K. Characteristics of bread prepared from wheat flours blended with various kind of newly developed rice flours. Journal of Food Science, Barking, v. 74, n. 3, p. E121-E130, 2009. http://dx.doi.org/10.1111/j.17503841.2009.01088.x. PMid:19397717.

NATIONAL ACADEMY OF SCIENCES - NAS. Dietary reference intakes: recommended intakes for individuals, vitamins, .minerals and macronutrients. Washington, 2014. Disponible: <http//:nap. edu/openbook,2000>. Acceso: 15 oct. 2015

RIO GRANDE DO SUL. Governo do Estado. Secretaria da Coordenação e Planejamento. Arroz. Porto Alegre, 2011. Disponible: <http://www.scp.rs.gov.br>. Acceso: 05 dic. 2011.

SANZ-PENELLA, J. M.; COLLAR, C.; HAROS, M. Effect of wheat bran and enzyme addition on dough functional performance and phytic acid levels in bread. Journal of Cereal Science, London, v. 48, n. 3, p. 715-721, 2008. http://dx.doi.org/10.1016/j. jcs.2008.03.006.

SANZ-PENELLA, J. M.; TAMAYO RAMOS, J. A.; SANZ, Y.; HAROS, M. Phytate reduction in bran-enriched bread by phytase-producing Bifidobacteria. Journal of Agricultural and Food Chemistry, Washington, v. 57, n. 21, p. 10239-10244, 2009. http://dx.doi. org/10.1021/jf9023678. PMid:19817458.

SANZ-PENELLA, J. M.; WRONKOWSKA, M.; SORAL-SMIETANA, M.; COLLAR, C.; HAROS, M. Impact of the addition of resistant starch from modified pea starch on dough and bread performance. European Food Research and Technology, New York, v. 231, n. 4, p. 499-508, 2010. http://dx.doi.org/10.1007/s00217-010-1294-7.

SHI, Y.; AZAB, A. N.; THOMPSON, M. N.; GREENBERG, M. L. Inositol phosphates and phoisphoinositides in health and disease. Sub-cellular Biochemistry, London, v. 39, p. 265-292, 2006. http://dx.doi.org/10.1007/0-387-27600-9_11. PMid:17121279. 